\title{
An OpenEHR Template with the Integrated German LOINC Terms
}

\author{
Abdul-Mateen RAJPUT ${ }^{\mathrm{a}, 1}$ and Samer ALKARKOUKLY ${ }^{\mathrm{a}}$ \\ ${ }^{a}$ Universitätsklinikum Köln, Kerpener Str. 62, 50937 Köln, Germany
}

\begin{abstract}
An OpenEHR template based on LOINC terms in German language (LOINC-DE) has been created for the structured clinical data capture. The resulting template includes all terms available in LOINC-DE, which can be selected from the drop-down menu for clinical data capture. The template can be used as an independent laboratory form or it can be customized for local needs. This approach presents the possibility to include terminologies in EHR when capturing patient data.
\end{abstract}

Keywords. OpenEHR, LOINC, Semantic Interoperability, HiGHmed

\section{Introduction}

OpenEHR is a vendor-independent healthcare information modeling specification which enables modelling of interoperable Electronic Health Records (EHRs). It provides opensource platform for the exchange of clinical data. Since its inception, it has been used widely across the globe[1-3]. It is based on two-level modelling: Reference Model and Archetype Model, terminology binding is considered as a third major model of OpenEHR [2]. LOINC (Logical Observation Identifiers Names and Codes) terminology facilitates the use of medical data in clinical care and research by providing a set of universal names and codes for a variety of laboratory and clinical test results. In this work, an OpenEHR template based on LOINC terms in German language (LOINC-DE) has been created for the structured clinical data capture. This template provides an easyto-use interface without compromising the data integrity of clinical data registries.

\section{Methods}

The LOINC-DE was extracted from Unified Medical Language System [4] by using the method described in the paper [5]. The naming convention of LOINC-DE is selfdescriptive e.g. Haemoglobin is written as "Hämoglobin:MKnz:Pkt:BlutNaV:Qn:", which includes MKnz for Property, Pkt for Time, BlutNaV for System and Qn for Scale. Only concept names e.g. Hämoglobin and codes were chosen to be included in the template. After getting all the concepts from LOINC-DE, an OpenEHR archetype containing only one "cluster" element was developed by using Archetype Editor [6]. Then the template was generated from the archetype. Ocean Informatics Template Designer tool [7] was used to import all LOINC-DE terminology concepts into a

${ }^{1}$ Abdul-Mateen RAJPUT, Universitätsklinikum Köln, Kerpener Str. 62, 50937 Köln, Germany ; E-mail: Abdul.mateen@uni-koeln.de. 
Valueset of the template by selecting the option "Load value list from text file" from the Properties Browser window. Please note that the function requires CSV file with three attributes i.e. Terminology, Code, and Value.

\section{Results}

The resulting template includes all terms available in LOINC-DE, which can be selected from the drop-down menu (figure 1) for clinical data capture. This allows users to specify exactly which concept needs to be used in the reports, assuring a unified and standardized concept name in all different medical reports, improving the semantic interoperability. In addition, the Template contains the LOINC code associated with a concept name, thus making it ideal for any further data processing operations e.g. finding synonyms in another language or retrieving concept associated metadata from LOINC database.
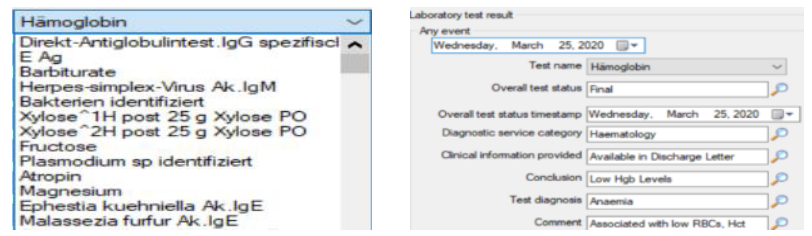

Figure 1. The complete LOINC-DE Terminology as a drop-down menu (left) and an example of a filled form with the concepts from LOINC-DE in the field "Test name" (right).

The template can be used as an independent laboratory form as well as it can be customized for local needs. Using this template will also save time and efforts of users as they do not have to search for the standard terms as well as LOINC codes separately. The Template is publicly available at: https://github.com/abdulmateenraj/OpenEHR.

\section{Discussion and Conclusion}

The approach presented in this paper shows the possibility to include terminologies in OpenEHR medical forms when capturing patient data. This simple idea can remove ambiguities and improve the semantic interoperability among different clinical systems significantly. The similar approach can be used for other clinical terminologies which are crucial for error-free clinical data capture e.g. ICD-10 or SNOMED-CT.

\section{Reference}

[1] Maia TA, et al. Archetype Development Process: A Case Study of Support Interoperability among Electronic Health Record in the State of Minas Gerais, Brazil. J Med Syst 2019; 43:57.

[2] Min L, Tian Q, Lu X, et al. Modeling EHR with the openEHR approach: an exploratory study in China. BMC Medical Informatics and Decision Making 2018; 18: 75.

[3] Mascia C, et al. The openEHR Genomics Project. Stud Health Technol Inform 2020; 270: 443-447.

[4] UMLS Terminology Services -- Home, https://uts.nlm.nih.gov/home.html (accessed 30 March 2020).

[5] Rajput AM, Gurulingappa H. Semi-automatic Approach for Ontology Enrichment Using UMLS. Procedia Computer Science 2013; 23: 78-83.

[6] Archetype Editor Home, https://www.openehr.org/downloads/archetypeeditor/home (accessed Mar 2021).

[7] Template Designer | Ocean Health Systems, http://www.oceanhealthsystems.com/products/templatedesigner (accessed 29 March 2020). 